\title{
A QUALIDADE DA EDUCAÇÃO NA COLÔMBIA
}

\author{
LA CALIDAD DE LA EDUCACIÓN EN COLOMBIA ${ }^{1}$
}

EDUCATION QUALITY IN COLOMBIA

\author{
John Alexis Rengifo CARPINTERO ${ }^{2}$ \\ Carmen Helena Díaz CAICEDO ${ }^{3}$
}

RESUMO: Este discurso reflexivo mergulha em termos genealógicos, a categoria políticoadministrativa da qualidade da educação. O contexto: Colômbia. O problema: a consideração comercial da qualidade da educação como o aumento dos números em governos campanhas governamentais. Ele vendeu a ideia de que a educação de qualidade corporativa tem a ver com a infra-estrutura cada vez maior, e cobertura de treinamento. Em nenhum momento ele veio a hipótese de que o problema da educação no país não é uma questão de quantidade, mas de qualidade. Portanto, este trabalho, de um lado, problematizar a noção de qualidade do ensino; por outro lado, propõe-se a falar de um cross-training proposta entre a família, escola, comunidade e do Estado.

PALAVRAS-CHAVE: Educação de qualidade. Projeto de formação. Homogeneizaçãoracional.

RESUMEN: Este discurrir reflexivo indaga, en términos genealógicos, por la categoría político-administrativa de la calidad de la educación. El contexto: Colombia. El problema: la consideración mercantil de la calidad educativa como aumento de cifras en las campañas gubernamentales de los gobiernos de turno. Se vende a los societarios la idea de que una educación de calidad tiene que ver con el aumento de infraestructura, cobertura y capacitaciones. En ningún momento se ha llegado a plantear la hipótesis de que el problema educativo en el país no es una cuestión de cantidad sino de cualidad. Por ello, este trabajo, de un lado, problematiza la noción de calidad educativa; de otro lado, propone hablar de un proyecto de formación intersectorial entre: la familia, la escuela, la comunidad y el Estado.

\footnotetext{
${ }^{1}$ Este artículo es un avance del proyecto de investigación titulado El canto: una práctica pedagógica de resistencia y liberación en las comunidades afrodescendientes del pacífico colombiano, gracias al apoyo de la Universidad Santiago de Cali, y con la participación de la Asociación Eslabón Cultural, la Fundación Investigación Creativos con la comunidad de cantaoras del grupo Integración Pacífico de la comuna trece de la ciudad de Cali.

${ }^{2}$ Universidad Santiago de Cali (USC), Cali - Colômbia. Profesor e investigador. Profesor Investigador de la Universidad ESAP. Magister en filosofía de la Universidad del Valle. Director de investigación de la Asociación Eslabón Cultural bajo el modelo de alfabetización crítica. Líneas de investigación: pedagogía crítica, filosofía crítica, historia comparada de las religiones y antropología cultural. ORCID: <http://orcid.org/0000-0001-75271241>.Correo: jalexrecar@yahoo.es

${ }^{3}$ Universidad Santiago de Cali (USC), Cali - Colômbia. Profesora de la institución educativa Divino Salvador. Profesora por prestación de servicios de la Universidad Santiago de Cali. Directora de la Asociación Eslabón Cultural. Líneas de Investigación: Teatro del oprimido, Antropología teatral y Pedagogía crítica. Profesional en Teatro y Danza del Instituto Popular de Cultura. ORCID: <https://orcid.org/0000-0001-5554-7335>. Correo: distriarte@ hotmail.com.
} 
PALABRAS CLAVE: Calidad educativa. Proyecto de formación. Homogenización-racional.

ABSTRACT: This reflexive discourse investigates, in genealogical terms, the politicaladministrative category of the quality of education. The context: Colombia. The problem: the commercial consideration of educational quality as an increase in figures in the government campaigns of the governments on duty. The idea that a quality education has to do with the increase of infrastructure, coverage and training is sold to the company. At no point has the hypothesis been reached that the educational problem in the country is not a question of quantity but of quality. Therefore, this work, on the one hand, problematizes the notion of educational quality; On the other hand, he proposes to speak of an intersectoral training project between: the family, the school, the community and the State.

KEYWORDS: Educational quality. Training Project. Homogenization-rational.

\section{La comedia}

Entre los meses de mayo y junio del año 2014, la ciudadanía colombiana fue direccionada por los medios de comunicación hacia una terrible tensión social en torno a una comedia: las elecciones presidenciales.

La actual campaña presidencial de Colombia, donde este domingo habrá elecciones, parecería ser, según analistas consultados por BBC Mundo, la más sucia de los últimos tiempos [...] este país, donde un expresidente fue acusado de recibir dineros de la mafia en su campaña, donde varios congresistas fueron a la cárcel por el mismo motivo, y donde muchos más han sido condenados por tener nexos con los paramilitares de derecha, no había visto episodios similares como los de los últimos días (SALAZAR, 2014, p. 1).

Los comediantes: el presidente de turno el señor Juan Manuel Santos, y su opositor, el señor Oscar Iván Zuluaga. El primero, abanderado de un discurso pacifista: proceso de paz con la Guerrilla de las (FARC). En cuya lógica proselitista, las supuestas altas cifras en los temas de empleabilidad, vivienda y educación gratuita, eran la punta de lanza de un discurso demagógico reeleccionista. De otro lado, el segundo, un político hecho a la imagen y la medida de los politiqueros de antaño, poseedor de una actitud y discurso guerrerista, monopolista, elitista y poco autónomo, trataba de caminar sobre los pétalos rojos de un camino ya ensombrecido, dejados por la carrera armamentista de un payasito, ni siquiera bufón porque no le cabe el apelativo, su padrino y mentor político, el principal titiritero y comediante de Colombia, Álvaro Uribe Vélez. Este último, se autodefinía como el presidente de la educación, de los cafeteros, de las fuerzas militares, de los campesinos, en otras palabras, le faltaba muy poco para ser Dios. 
Ante dicha tensión social, maximizada por los monopolios comerciales de las dos principales familias acaudaladas del país: los Santo Domingo y los Ardila Lule, empoderadas de los medios de comunicación más vendidos del país: (RCN) y (CARACOL), los ciudadanos debían elegir entre un mal gobierno y otro que parecía peor. Los societarios se debatían entre qué propuesta gubernamental era menos mala.

La malignidad y teatralidad de tales campañas políticas se evidenciaba precisamente por: uno, la implementación de una propaganda política desleal; dos, la falta de propuestas políticas, económicas y sociales serias, reales y duraderas que partieran de las condiciones de vida de los ciudadanos, que pudieran mostrar las salidas plausibles a las problemáticas que éstos enfrentaban: altos niveles de desempleo y crecimiento del empleo informal, aumento cuantitativo en Bandas Criminales al interior de las ciudades que deteriora el tejido social de estas, ninguna política pública sobre el aumento desmedido en el crecimiento demográfico en los sectores marginales de las ciudades, escazas propuestas pedagógicas que solucionaran la situación de matoneo, prostitución y drogadicción al interior de las instituciones académicas, ninguna propuesta que solucionara el problema del aumento en el flujo vehicular en todas las ciudades de Colombia etc. Tres, sus cortas, escazas y falaces propuestas sobre el mejoramiento de la calidad de la educación en Colombia, que se constituyó en tema central de una y otra campaña debido a un acontecimiento social específico: el último lugar ocupado por el país en las pruebas (Pisa). Lo que instituyó de una vez y para siempre las supuestas desventajas académicas en que se encuentran los jóvenes de 15 años de edad, de las instituciones académicas privadas y oficiales colombianas, ante sus contemporáneos de China, Finlandia o Canadá. Sin embargo, lo que los medios no dicen es que tal prueba es realizada por la Organización para la Cooperación y el desarrollo Económico (OCDE), bajo un único rasero: el desarrollo de competencias laborales internacionales. Lo que de una vez instituye un tipo de educación específica: un modelo de educación mundial para el trabajo, el desarrollo económico de los pueblos, la formación cuantitativa de mano de obra barata.

\section{¿Cantidad o cualidad educativa?}

Este escrito no es una empresa sobre la caricaturesca política que se da en nuestro país, donde se hace de la paz un juego político, de la justicia social una cifra estadística y de la educación una estrategia de campaña. No, este escrito no es sobre este tema, sino sobre cómo estos comediantes han concebido el tema más relevante, central y necesario en el desarrollo de toda vida orgánica y de todo pueblo, la educación (NIETSZCHE, 2000). Específicamente, la 
categoría conceptual administrativa: calidad de la educación. En nuestro país, desde hace más de dos décadas, la calidad de la educación parece ser un tema de administrativo de mercado: implementación sistemática de infraestructura (hacer más escuelas, más colegios, más universidades), aumento sistemático en la cobertura educativa (más estudiantes por salón, por escuela, por colegio y por universidad, amotinamiento educativo), reproducción en serie de más tecno-docentes (más profesores de carrera o no, poco importa que no sean pedagogos, para más colegios), en suma, en Colombia se ha creído que la educación es un tema económico: aumento de cifras que midan la inserción del estudiante primero a la escuela, segundo al colegio y tercero a la universidad. Competencias del educador en manejo de Tics, dominio de una segunda lengua (preferiblemente el Ingles) y eficiencia en el relleno de formatos, para consolidar una educación con altos estándares de "calidad". Sin embargo, en Colombia, ni los gobernantes de turno, ni los rectores asalariados, ni los padres de familia ocupados en sus quehaceres laborales ${ }^{4}$ se han dado cuenta que la calidad en la educación no es un tema de cantidad sino de cualidad. Si, señores y señoras, la educación es un tema de cualidad y no de cantidad.

El concepto de calidad educativa es, genealógicamente hablando (NIETZSCHE, 1989), un acontecer neoliberal, propio del modelo de producción capitalista, surgido a mitad del siglo XX. El modelo de control de la producción: medición, parametrización, estandarización, de las mercancías (GIROUX, 2004) el cálculo de beneficios, fue trasladado al ámbito educativo para poder medir, regular, vigilar, disciplinar el proceso (FOUCAULT, 1996), y, con ello, a los agentes activos del mismo: el educador, el educando (ZULUAGA, 1996) el contenido curricular (QUICENO; PEÑALOSA, 2014) la interacción con el entorno. En términos de maximización de recursos versus minimización de costos, gastos. En Colombia la fuerza ilocutiva de tal discurso (HABERMAS, 1999) aparece después de los años 90. Coincide, claramente, con la política de la apertura económica del gobierno del presidente Cesar Gaviria. Lentamente la fuerza enunciativa del discurso se trasformó en fuerza perlocusionaria (AUSTIN, 1955) no reflexionada, no problematizada, no cuestionable que legitimó tal categoría como tema central en educación. En los anales de la historia la categoría, calidad, no aparece como tema central en ninguna reflexión pedagógica.

Este escrito inicia, entonces, con una pequeña consideración, a modo de pregunta, sobre la educación en el contexto de un mundo global, para plantear de lleno un problema real ¿puede el aumento de infraestructura, asignaturas, profesores, el mecanismo que asegure la calidad de la educación en una nación, claramente sometida a un contexto global? Más, si con tal

\footnotetext{
${ }^{4}$ Insertos en el mundo de un sedentarismo-nómada tecno-científico.
} 
consideración sólo se presupone una cuestión ya existente: un proceso educativo de baja, mediana, o nula calidad, el problema real en la educación actual, global, ciber-espacial, tecnocientífica, racional-homogenizante, ha de ser el siguiente: ¿cómo educar hoy, a quién no quiere aprender?, o lo que es lo mismo ¿se puede enseñar a quién no está motivado en aprender? Y si deseáramos poner el dedo en la llaga, ¿hoy, y sólo hoy, se puede enseñar?

Problema que inmediatamente evidencia de un modo real el desplazamiento que debería hacerse sobre el a priori social: calidad educativa, por una cuestión a una más central: cualidad educativa. Lo que tornaría gris el concepto en discusión.

El problema anteriormente expuesto manifiesta la coyuntura, el vacío conceptual que existe a nivel social, pedagógico, sobre la enseñanza (ZULUAGA, 1996) no sólo en Colombia, sino en el mundo global. Situación que surge por un doble tema: a) la falta de motivación del educando ante ningún proyecto real, serio, de formación escolar, tanto de parte de las instituciones académicas, como de las prácticas pedagógicas de los educadores (COREA; LEWKOWICZ, 1995); b) el devenir de un mundo dinámico tecno-científico y ciber-espacial carente de toda motivación existencial por un proyecto de vida plena (RENGIFO; PINILLO; DÍAZ, 2016).

Nuevos acontecimientos producidos en los últimos años han confirmado en gran medida nuestra sospecha de que la locura que nos rodea funciona dentro de prácticas discursivas más amplias. Hemos denominado "conocimiento de los medios de comunicación" al resultado de tales prácticas (MCLAREN, 1991, p. 139).

A no ser que consideremos como un proyecto serio los (PEI), y entonces, ¿para qué seguir gastando tinta sobre algo que es funcional? Al parecer, si en Colombia la calidad en la educación es un tema central es porque en la estructura académica tal y cual como se ha dado el proceso de enseñanza-aprendizaje en la escuela, colegios y universidades, seriamente, no ha sido más que una terrible broma, un fracaso rotundo.

No obstante, centrémonos en la consideración político-administrativa de la calidad en la educación. La calidad en la educación en Colombia, claramente, no pasa por ser un tema de mercado. La educación no es un tema de mercado, de aumento de infraestructura, de aumento en el porcentaje de inserción de los estudiantes a la escuela, ni de incremento en la planta profesoral, es un tema esencialmente de cualidad en el proceso motivacional y actitudinal de enseñanza-aprendizaje entre educador y educando (FREIRE, 1971). Para demostrarlo atendamos a la siguiente evidencia histórica. 


\section{La evidencia histórica: una genealogía del presente cultural}

Entre los años 80 y 90 en la ciudad de Cali Colombia existían en promedio 10 pandillas juveniles con un promedio de edad de 21 años, que se ubicaban en los sectores más vulnerables de la ciudad. Después del año 2000, entre el 2005 y el 2013 el número de pandillas juveniles paso de 10 a 137 con un promedio de edad de 17 años, ubicadas no sólo en sectores marginales, sino en sectores de los que nunca se habían tenido datos, barrios de clase media y alta. Los datos arrojados por la Asociación Eslabón Cultural ${ }^{5}$ muestran que en barrios como Lagos I de la comuna trece, en cada cuadra existe una pandilla de niños de dicados al robo, la extorción y el micro tráfico de drogas. Más aún, los estudios de la Asociación muestran también en los barrios de estrato social alto, el número de pandillas se ha incrementado, con el mismo modelo delincuencial; lo que indica que el pandillismo en Cali no es una cuestión que sólo se da en sectores marginales de la ciudad, sino que este fenómeno ha permeado toda la estructura social de la misma. Lo curioso de esto es lo siguiente. Entre el mismo periodo histórico, años 80-90, el número aproximado de escuelas (públicas y privadas), colegios, universidades, iglesias y organizaciones no gubernamentales era menor a 100, con excepción de las (ONG) y universidades que no superaban los 10 por cada una. Después del 2005 las escuelas y colegios superan los 300, las universidades llegan a las 10 y los (ONG) superan las 400. Lo que quiere decir lo siguiente: si la educación es una cuestión de cantidad el aumento en el número de escuelas, colegios y universidades después de los años 90 hubiesen tendido a disminuir el número de pandillas en la ciudad, a tal punto que era inminente su desaparición; pero la evidencia histórica demuestra lo contrario. La realidad mostró que por el contrario con el aumento de más escuelas, colegios, universidades, confesiones religiosas y ONG el número de pandillas de jóvenes menores de edad aumento en un $1.200 \%$, esto quiere decir que la educación no es un problema de cantidad, sino de cualidad y pone de manifiesto la reacción de niños y jóvenes ante ninguna propuesta vinculante e intersectorial en las políticas públicas en el tema de educación. Pues la familia cree que cumple con moralizar, la escuela con instruir e informar, la comunidad con divertir y el Estado con insertar. Pero en ningún momento han pensado que el niño y el joven no se sienten leídos como una parte constitutiva de un todo orgánico (NIETZSCHE, 2000), dentro de un sistema que los considera átomos en calidad de ciudadanos, actores estudiantes. Por el contrario éstos se sienten como una isla en medio de un océano de información, información y más información. Donde la familia lo informa sobre lo que debe

${ }^{5}$ La Asociación Eslabón Cultural es una organización sin ánimo de lucro que realiza trabajo social, a nivel artístico, cultural e investigativo, basado en el modelo de la pedagogía crítica, en las comunas trece, quince y veintiuno de la ciudad de Cali. 
ser, la escuela lo instruye e informa sobre lo que debe aprender, la comunidad lo alienta, instruye e informa sobre lo que debe tener (LIPOVETSKY, 1986) y el Estado lo considera, informa e instruye sobre lo que necesita saber hacer, empleado, obrero, mano de obra barata, aunque calificada (RENGIFO; PINILLO; DÍAZ, 2016a).

La cualidad educativa implica una cosa distinta a aumentar cifras, no es sólo números que midan el impacto de un gobierno sobre la población. La calidad en la educación en Colombia seguirá el mismo rumbo, la mediocridad, mientras se la siga considerando en términos financieros. La calidad de un sistema educativo es un problema de cualidad estructural entre sus partes constitutivas: la familia, la escuela, la comunidad y el Estado (BORDA, 2015) lo que implica una relación intersectorial entre estos. Pero, el sistema educativo en Colombia seguirá fracasando mientras no se den dos cosas: una, un diálogo reciproco entre estas partes constitutivas tendente e un proyecto conjunto de formación, y que más allá del centro sea capaz de pensarse también, dese las periferias, la indígena, la negra (2015). Dos, una consideración profunda sobre la relación constitutiva que se da entre los actores sociales participes en el acto educativo, el educador y el educando (FREIRE, 1971) insertos en el entramado vivencial de un mundo de la vida (HABERMAS, 1999) contextual específico. De modo tal que la educación no sea más un proceso de homogenización racional (1971) sobre el trasfondo conceptual de un mundo tecno-científico como garante epistemológico de un proyecto ilustrado (RENGIFO, 2014) basado en la idea de un progreso social, siempre económico, civilizador ique no se han dado cuenta que ha fracasado!

¿Qué proyecto de educación real se ha discutido entre la escuela y la comunidad?, ¿en qué momento la escuela se ha sentado a discutir con la familia un proyecto de formación conjunto?, ¿qué diálogos se han dado entre la escuela, la familia y la comunidad para disminuir los índices de pandillaje y plantear un proyecto de formación conjunto, centrado en la relación educador-educando en un proceso de enseñanza-aprendizaje contextual?, ¿en qué momento se ha dado un diálogo Estado-comunidad-escuela proyecto de formación?

\section{Otro acontecimento}

Los diálogos de la Habana entre gobierno y FARC, arrojaron en octubre del 2016 unos acuerdos cuyo desconocimiento, de parte de la gran mayoría de los colombianos, como la manipulación demagógica, de quienes los “conocían", dieron como resultado el fracaso de un plebiscito por el sí, con el que se pretendía legitimar estos acudiendo ante la voluntad del pueblo. El terrible resultado, lo inesperado, la derrota del mismo por el no 51\%. Acontecimiento 
que dejó como evidencia un mapa político en el que claramente las regiones que no han sufrido el flagelo de la violencia guerrillera, o paramilitar, de un modo directo, pero que, se han caracterizado por ser las regiones petroleras: Antioquia, Caquetá, Risaralda, Llanos, entre otros, optaron por el no a expensas del sufrimiento del resto de pueblos y municipios olvidados por los gobiernos de turno, pero que han de servir para ganar votos.

En uno u otro caso, el resultado era el mismo: la manipulación del pueblo, como ha sucedido a lo largo y ancho de la historia mundial, y el beneficio de los poderosos. En dicho acontecimiento socio-político algo, nuevamente, fue olvidado: la educación como tema central en el desarrollo humano de una nación (PLATÓN, 1979). Eso sí, se hizo, nuevamente, de los educadores, peones en el juego del ajedrez político. ¿Y la educación? Cumplió una función: un campo de batalla, movido por las maquinarias políticas de uno u otro partido, para hacer proselitismo, demagogia académica al servicio de intereses políticos de las élites. Los acuerdos se desconocían pero se enunciaban, parafraseaban, "argumentaban", etc. Dos cosas quedaron claras: la primera, la división del país en términos sociales, políticos, económicos e ideológicos. La segunda, la manipulación histórica, que se ha hecho de la educación como de los educadores, en tanto en cuanto, actores serviles en el juego de los intereses políticos de los que ostentan el poder financiero. El terrible resultado: una educación sin formación.

\section{Un proyecto de formación intersectorial}

Lo anterior quiere decir que en Colombia se debe plantear una discusión seria sobre la dimensión social que permite tales relaciones, la pedagogía. Es decir, se le debe devolver el estatuto epistemológico al saber pedagógico en su acción formadora (ZULUAGA, 1996). Tal esfuerzo implicaría ¿qué educando es el que socialmente se desea formar?, ¿mano de obra barata al servicio de las multinacionales?, y entonces se hace del acto educativo un proceso de tecnificación del proceso de enseñanza-aprendizaje (ALTHUSER, 1988), y se les vuelve a los estudiantes obreros capacitados para reproducir una destreza específica en un entramado empresarial concreto (MARCUSE, 1993). O por el contrario se desean ¿ciudadanos para un mundo globalizado? Y entonces, se hace de las finanzas el tema central de la educación, a tal punto que ésta sólo actúa como garante y reproductora del mundo tecno-científico comercial y ciber-espacial. Y el economista pasa a ser el estereotipo de hombre "formado". Bueno, y ¿qué maestro se desea tener? ¿Eruditos del conocimiento, profesionales del saber, tecno-educadores al servicio de monopolios financieros? Entonces se hace de la educación la reproducción en serie del ideal de un mundo feliz (HUXLEY, 2014) bajo el paradigma de las Tic, la red, y la tv. 
De nuevo el ideal ilustrado vuelve a aparecer. Pero, ¿y cómo se debe enseñar?, ¿bajo la perpetuación de una pedagogía mnemotécnica de antaño, aunque funcional?, o por el contrario ¿bajo la incursión de modelos pedagógicos nuevos centrados en procesos de psicologización del acto educativo (ALTHUSER, 1970) donde el maestro debe centrarse única y exclusivamente en comprender el mundo dinámico, subjetivo y evanescente del niño (ROUSSEAU, 1978), pero bajo los mismos espacios cerrados y bajo en horizonte escolástico de la calificación, la evaluación y la nota (QUICENO; PEÑALOSA, 2014)? Formas, modelos y prácticas que en su conjunto han sido, y seguirán siendo un fracaso. La psicologización del acto educativo ha arrojado como resultado la pérdida de autoridad del padre, la madre y el maestro, y el aumento indiscriminado del pandillaje (RENGIFO; DÍAZ; PINILLO, 2016a). La Falta de diálogo serio entre comunidad, Estado, escuela y familia ha arrojado como resultado el conocimiento parcializado de la educación en ciencias de la educación, centradas en un modelo de educación económico: educación para el desarrollo.

La calidad en la educación tiene que ver con dos procesos educativos paralelos pero que deberían ser opuestos. El primero, la educación cultural-contextual (BORDA, 2004). Esta forma de educación es la encargada de transmitir un acervo de saber cultural al conjunto de la sociedad. En el mundo posmoderno es el saber tecno-científico, bajo el horizonte de un mundo ciber-espacial, el que se autolegitima como saber a ser deseado y reproducido por las prácticas sociales del conjunto de los societarios. El segundo, la educación para la formación planeada, medida de un tipo de hombre específico. Este es el tema central de la dimensión educativa, del saber pedagógico en tanto institución social. Esta desarrolla el anhelo, el deseo de formar a un hombre para... Un fin. Es un plan, un proyecto social de formación.

Entre estas dos formas de educación debería haber un espacio de confrontación, de tención de coyuntura, pero no sucede así. En el mundo actual el segundo sistema no problematiza el primero, antes bien lo reproduce felizmente, por lo que todos los errores y horrores del primero son introducidos en el segundo en forma de práctica pedagógica constante. Por lo que es en la segunda en donde hablamos de falta de calidad en la educación, así en la primer forma de educación se acuda a un mundo carente de sentido caracterizado por un vacío constante (LIPOVETSKY, 1986). Es común ver en las prácticas pedagógicas de los educadores contemporáneos a través de las instituciones de la reproducción del saber social tecnocientífico, la apuesta por un saber memorístico, mecánico y poco crítico. La tarea, la "investigación" de un tema para la casa vía internet, el examen de memoria siguen siendo los recursos didácticos de educadores en todo el país. Y a eso se le llama enseñar. A la reproducción de un modelo social en donde el sí mismo, el otro y la naturaleza han sido reducidos a su mínima 
expresión, su existencia como ciber-seres. En las políticas educativas de todas las universidades del país la inserción de las Tic, al aula de clase, se ha impuesto como una necesidad "didáctica", a tal punto que el profesor que no lo haga será tachado de incompetente, pues es ese el saber que pide, exige, requiere la sociedad de hoy. Lo que demuestra que la misión de educar que le correspondía a estos sacros santos lugares ha dejado de serlo: uso de la facultad de juzgar, el juicio crítico en acción de co-construcción de un mundo lleno de sentido. Por lo que la calidad de la educación seguirá siendo un tema de mercado: aumento en número de lugares, personas y programas. $\mathrm{Si}$, a eso se le ha llamado en Colombia "Calidad educativa", aunque la descomposición del tejido social muestre lo contrario.

Ahora bien, como no existe ningún proyecto de formación real de parte de las instituciones académicas, ni de sus educadores, ya que los primeros tan sólo quieren recursos económicos para poseer capital financiero que les permita una mejor calidad de vida para consumir, entonces se hace de la cobertura educativa un negocio; mientras que los segundos sólo buscan llenar la barriga, consumir entretenimiento y pagar deudas. Los educando acuden a las instituciones de educación a consumir paquetes de información, propios de las disciplinas escolares (física, química, biología, historia, filosofía, etc) de un modo generalmente pasivo, aburrido y evanescente. Pues, éstos se hallan divididos entre un mundo afectivo-familiar moralizador caótico, barrial-emocional complejo, tribal-afectivo instituyente y académicodisciplinar informador, por lo que la dispersión, la hiperquinesis y banalidad se hacen presentes en su acción de aprendizaje.

Hoy se sufre por saturación, porque todo el sensorio ha sido ocupado. El problema hoy es que el mundo interno no puede constituirse. Las dos figuras de la saturación son los hiperkinéticos y los aburridos, dos efectos distintos y complementarios de la saturación mediática. Los adolescentes sufren, padecen el aburrimiento por desolación (COREA, 1995, p. 69).

El terrible resultado: niños, niñas y jóvenes fugados de la realidad, ocultos tras las redes sociales con sus semejantes tribales y autómatas de un ciber-mundo. La consecuencia: una educación pobre en contenido, procesos, didácticas y desarrollo del juicio crítico, falta de calidad educativa.

Lo anterior significa que, ante el mundo de la vida de los educando caracterizado por la acción dinámica de una cultura posmoderna, material, hedonista, consumista de tecnología e información, la escuela deviene como un espacio superfluo de aburrimiento, adoctrinamiento e individuación disciplinar. El educando percibe tal realidad, y lo que representa el poder instituido por el educador, como una realidad ajena a su ciber-mundo (RENGIFO; DIAZ; 
PINILLO, 2016b). Pues el mundo del flujo constante de información y de encuentro con los pares, con los iguales, con los miembros de la tribu, es comprendido como el espacio real de institución de los afectos verdaderos. El chat, la comunicación virtual se transforma en espacio de fuga y de encuentro con el igual que sobrepasa el espacio arquitectónico concreto de lo académico-formal-disciplinar. La escuela disciplina, el ciber-espacio libera, así piensa el joven de hoy. La escuela instruye sobre la historia de las ideas ya instituidas sin la participación del educando, por lo que se antepone al mundo del educando, por el contrario a través del ciberespacio el mundo se construye con los miembros de la tribu día a día y a este se le construye y reconstruye o se le hace desaparecer en un acto insignificante de broma. Así tal acto de coconstrucción de significado sea superfluo, este construye realidad: realidad superflua.

De ahí que, el paradigma comunicativo de estos agentes sociales sea la broma, el chiste, la acción social de vender la imagen de otro ridiculizado. El otro vuelto objeto de escarnio público se hace tema de conversación. La acción lingüística del uso de la facultad crítica es superada por la acción mediática de generar el efecto, socialmente deseable, de reírse de algo, o de la tragedia de alguien (RENGIFO, 2018). En tal subjetividad del educando contemporáneo, la palabra como acto de vinculo y encuentro sobre un tema en el mundo de la vida (HABERMAS, 1999) desaparece ante el influjo comercial, mediático, estimulante de una acción narrativa vuelta frase, monosílabo o imagen, que produzca una terrible carcajada o un terrible espanto. El morbo de reírse o espantarse de la tragedia de un otro cualquiera, se hace acción pedagógica de esa primera forma de educación, la cultural. Por lo tanto, la educación al interior de los espacios educativos hechos para instituir el juicio crítico se hacen superfluos, carentes de sentido y de significado. El mundo del ciber-espacio a través de la red, instituye un mundo a ser aprendido o bien como carente de sentido y esencialmente trágico, y es por esta razón que ha de ser ridiculizado y vaciado de todo contenido positivo; o bien, como un mundo del que hay que vivir, hedonistamente, en su futilidad existencial, ante ningún proyecto de formación social real.

De ahí que ante ningún proyecto de formación, social real, el mundo ciber-especial deviene como acción social de realidad fundante de sentido para el educando, así este sea corto en contenido formativo. Realidad superflua que no deja de disfrazar la actitud de pereza, hiperquinesia y aburrimiento del educando. Si no se les puede enseñar es porque no desean aprender. Ya que entre la educación cultural y la institucional se da una terrible relación de codepencia respecto al fin: informar y disciplinar mediante paquetes tecnológicos, al mismo tiempo que se presenta una situación de exclusión respecto a las prácticas de los estudiantes. Puesto que, la segunda forma de educación no reproduce fielmente la dinámica audio-visual de 
la primera. Por lo que la escuela termina siendo un espacio de tedio, encuentro ritual de cortejo y desarticulación del mundo de la vida privado dinámico audio-visual, frente al mundo público aburrido e institucional-formal.

Por el contrario, si se da un diálogo intersectorial entre la familia, la comunidad, la escuela y el Estado en un proceso de revisión de la relación educador-educando (RODRÍGUEZ, 2014) que ha de entender que la educación es un proceso relacional que implica poner en juego un conjunto de saberes, de parte del maestro, en relación con el mundo contextual del educando, que también pone sobre la mesa sus saberes barriales, tribales, comunales, vecinales, profesionales en un acto de encuentro común, en el que se dan dos situaciones: la primera, se deconstruye (DERRIDA, 1986) la realidad que no potencie la sensibilidad creativa del educando en todas sus dimensiones; la segunda, se co-construyen nuevas realidades positivas en torno a las múltiples dimensiones del ser. Lo que implica reevaluar las actuales prácticas pedagógicas disicplinatorias, instruccioncitas y evaluativas. O sea, es descodificar la idea del estudiante de prepararse para ser evaluado en un tema, estudiar por la nota; y la idea del profesor, de ser él el único responsable y directamente encargado del proceso de enseñanza, cuando en su ayuda deben acudir todos los miembros de la comunidad. ¡El marrano es como lo crían! Lo que implica un agenciamiento constante el proceso de enseñanza-aprendizaje. A la escuela no se va a estudiar, se va a debatir, descubrir, problematizar, resignificar la realidad propia y ajena, se va protestar y denunciar los sistemas de exclusión, marginación y opresión sociales. El agenciamiento es la relación dialogada, acción del juicio crítico, en proceso de coconstrucción entre el educador y el educando. La escuela acontece como un espacio de problematización sobre la realidad. La evaluación surge como un medio que regula y motiva el proceso de inter-construcción basado en el principio de la auto-responsabilidad, pero jamás como un simple fin que mide competencias (capacidad de estar lleno de información para dar cuenta socialmente de ella).

\section{Conclusión}

En síntesis, la calidad de la educación en Colombia no puede seguir siendo un tema de mercado, de aumento en las cifras de alfabetización, de aumento en las cifras estadísticas de incremento en las políticas públicas gubernamentales de turno, para salvaguardar una estrategia política o un partido. Por ello, se debe plantear un ejercicio de vinculación intersectorial entre la familia, la escuela, la comunidad y el Estado para plantear un diálogo serio sobre un proyecto de formación conjunto, plenamente estructurado en el que cada parte ha de responder por las 
tareas sociales que le corresponden. De tal modo que se haga de la relación educador-educando en el proceso de enseñanza-aprendiza en torno a contextos sociales específicos un proyecto de formación real. Esto implicaría dos cosas: des psicologizar la educación y propender por procesos de formación social tendentes al desarrollo cualitativo de todas las dimensiones del ser, y no sólo a su perpetuación para una vida de obrero.

\section{REFERENCIAS}

ALTHUSSER, L. Louis Althusser, Freud y Lacan. Barcelona: Anagrama. 1970.

ALTHUSSER, L. Ideología y aparatos ideológicos del Estado. Buenos Aires: Nombre Falso. 1988.

AUSTIN, J. Cómo hacer cosas con palabras. Estados Unidos: Arsis. 1955.

BORDA, O. Una sociología sentipensante para América Latina. México: Siglo XXI. 2015.

DERRIDA, J. De la gramatología. México: Siglo XXI. 1986.

FOUCAULT, M. Vigilar y Castigar. Buenos Aires: Editorial siglo XXI. 1996.

FREIRE, P. La Pedagogía del Oprimido. Barcelona: Siglo XXI. 1971.

GIROUX, H. Teoría y Resistencia en Educación. Buenos Aires, Argentina: Editorial Siglo XXI. 2004.

HABERMAS, J. Teoria de la acción comunicativa. Madrid: Tauru. 1999.

HUXLEY, A. Un mundo feliz. México: Tauru. 2014.

LIPOVETSKY, G. La Era del Vacío. Barcelona: Anagrama. 1986.

MARCUSE, H. El hombre unidimensional. Barcelona: Planeta de Agostini. 1993.

MCLAREN, P. Pedagogía crítica, resistencia cultural y producción del deseo, Buenos Aires: Aique. 1991

NIETZSCHE, F. Genealogía de la moral. Barcelona: Tusquets. 1989.

NIETZSCHE, F. Schopenhauer como Educador. Barcelona: Tusquets. 2000.

PLATÓN. La República. Madrid: Edaf. 1979.

QUICENO, H.; PEÑALOSA. EI dispositivo de la evaluación: cartografía de la producción de conocimiento en el campo de la evaluación en Colombia, 1990-2014. Número 41, Universidad Pedagógica Nacional, Facultad de Educación, p. 45-61, 2014. 
RENGIFO, J. La educación como transmisión de la cultura: un conflicto entre innovación y tradición. Revista Diálogos Educativos, v. 27, p. 74-88, 2014.

RENGIFO, J.; PINILLO, M.; DÍAZ, H. La paradoja del espacio académico: disciplinar sin disciplina. Revista Colombiana de Educación, v. 70, p. 341-360, 2016 a.

RENGIFO, J.; PINILLO, M.; DÍAZ, H. El sedentarismo nómada. Madrid. Revista Internacional de Humanidades, v. 5, n. 1. $2016 \mathrm{~b}$.

RENGIFO, J. Una singularidad desencarnada: el problema de la enseñanza en la era digital. Revista Pedagogía y Saberes, v. 49, p. 213-224, 2018.

RODRÍGUEZ, E. Pedagogía crítica, una propuesta educativa para la transformación social. Reidocrea, v. 3, artículo 21, p. 147-17, 2014.

ROUSSEAU, J. Emilio o de la educación. Madrid: Edaf. 1978.

SALAZAR, H. La campaña electoral en Colombia: la más sucia de su historia. Bogotá: Mundo. 2014.

ZULUAGA, Pedagogía e historia. Medellín: Universidad de Antioquia. 1996.

\section{Como referenciar este artigo}

CARPINTERO, John Alexis Rengifo.; CAICEDO Carmen Helena Díaz. A qualidade da educação na Colômbia. Revista Ibero-Americana de Estudos em Educação, Araraquara, v. 14, n. 2, p. 326-339, abr./jun., 2019. E-ISSN: 1982-5587. DOI: 10.21723/riaee.v14i2.11735

Submetido em: 29/08/2018

Aprovado em: 10/11/2018 